\title{
Structure of Atomic Nuclei and Periodical Law of Mendeleev
}

Kholmanskiy A.S.

Laboratory of light engineering, Federal Scientific Agroengineering Center VIM, Moscow, Russia, vim@vim.ru

Correspondence: E-mail: allexhol@ya.ru, https://orcid.org/0000-0001-8738-0189

In the work, the nuclei structure was modeled by a system of shells consisting of combinations of spherical and annular vortices. Having connected the spin and the magnetic moments of the nuclei with the dynamics of the outer shell of the nucleus, we determined its type and carried out a semiempirical calculation of the parameters of its structure for stable and unstable nuclear isotopes in the sequence of the order number Z from 1 to 37. Comparison of the obtained nuclear sizes with the radii of the electron shells of atoms made it possible to reveal a definite correlation. On the basis of it, it was concluded that the periodic law of Mendeleyev can determine the patterns in the change in the structure of the atomic nucleus.

Keywords: nucleus structure; parameters of external shells; correlation of radii of nuclei and atoms; periodic law.

\section{INTRODUCTION}

D.I. Mendeleev not only substantiated the dependence of the chemical properties of elements on their mass number (A), but also predicted that «atoms of simple bodies are complex creatures formed by the addition of some even smaller parts» and «the periodic law does not threaten the future with destruction, but only superstructures and the development of being promised» [1]. With the discovery of the nuclear-electronic structure of the atom, the number $\mathrm{A}$ in the periodic law was replaced by the charge of the nucleus or the number of protons (electrons) in the nucleus $(\mathrm{Z})$. The number $\mathrm{Z}$ determines the atomic number in the periodic system of Mendeleyev. Due to the monotonicity of the changes in A and Z, they could not be directly related to the periodicity of the properties of the elements. This problem was solved only with the use of the formalism of quantum mechanics and the experimental data of atomic spectroscopy. The rules for quantizing the energy and population of electronic levels, as well as the concept of electronic orbitals and shells, have made it possible to relate the chemical properties of substances to the physical properties of the electronic structures of atoms and molecules. In this case, the periodicity of the properties of the elements was explained by the 
repetition of the scheme of constructing their external electron shell through certain periods in a sequence of numbers Z (Fig. 1).

The stability of the space-energy hierarchy of electron shells of an atom is due to the interaction between the nucleus and electrons. Moreover, taking into account the order of the ratio of the masses of the electron and the proton $\left(\sim 10^{-3}\right)$, we can conditionally assume that electrons label their orbitals with the lines of force of the atom's own electromagnetic field. The effect of the charge of the nucleus, its spin, and the magnetic moment on the shape and energy of this field is particularly clearly manifested in elements with small values of the number $\mathrm{Z}$. For example, the spin of a proton in a hydrogen atom largely determines the physicochemical properties of water [2,3]. The influence of the nucleus on the electronic levels of atoms manifests itself on their optical properties, which makes it possible to determine the nuclear spin, its magnetic and quadrupole moments, and the charge radius $\left(r_{e}\right)[4,5]$.

With all the obvious involvement of nuclei in the formation of electronic configurations of atoms, the dependence of their physical and chemical properties on the structure of nuclei in atomic physics is not taken into account. Guided by the laws of dialectics and using the rules of quantum mechanics, the author developed an algorithm for constructing and calculating the vortex model of the structures of elementary particles and nuclei [6-8]. Reliable experimental data in the algorithm play the role of boundary conditions. Thus, the vortex structures of the neutron, proton, and electron in the ground and excited states were calculated.

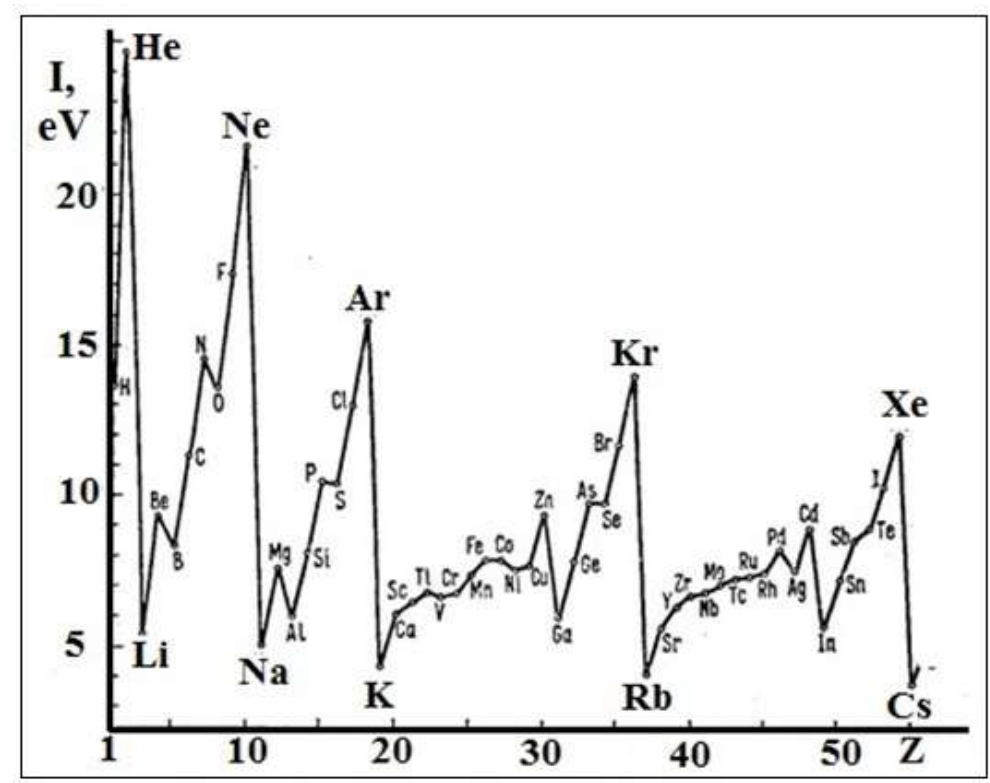

Figure 1. Dependences of the ionization potentials of atoms on the atomic number $(\mathrm{Z})$.

In this paper, the algorithm was used to calculate the vortex structures of stable and unstable isotopes of elements with $\mathrm{Z}$ from 1 to 37 in order to reveal correlations between the 
parameters of the nuclear structure and the physicochemical properties of the elements. A number of elements with $\mathrm{Z}$ from 1 to 37 include almost all macro and trace elements, the properties of which caused the emergence, and then the development of the terrestrial biosphere. In addition, this series of elements is sufficient to illustrate Mendeleyev's periodic law (Fig. 1).

\section{MATERIALS AND METHODS}

Data were calculated for the mass (m), the charge (q), the spin $(S)$, the magnetic $(\mu)$, and the quadrupole moment $(\mathrm{Q})$ of the nuclei from [9]. When choosing the optimal structure of the nucleus, the Q-sign was taken into account. In the formation of the magnetic-mass skeleton of the nuclear structure, three toroidal vortices-the spherical shell (Sh) and two annular vortices that are located inside (In) and outside (Ex) shells (Fig. 2). With these vortices, the electromagnetic energy flows forming the Kelvin oval and responsible for the electrical characteristics of the core are dynamically associated [7,8]. In Figure 2a, the Kelvin oval is indicated by a dashed line.

The thickness of the shell layer and the proper radius of the rings were considered much smaller than their radii $(r)$ and assumed $r_{\text {in }}=r_{\text {sh. }}$. The rules for assembling Sh, In and Ex from initial chiral vortices (energy forms, further - EF) are described in [6-8]. The nuclear structure included a system of embedded closed shells (CSH) formed from correlated pairs of protons $\mathrm{pp}^{*}$, further - (pp) and neutrons $n n^{*}$, further - $(\mathrm{nn})$ with inverse spins. The momentum $\left(\mathrm{L}_{\mathrm{i}}\right)$ of the nucleon elements in such pairs was zero, and the electric charge $\left(\mathrm{q}_{\mathrm{i}}\right)$ was summed. For CSH, $r_{e x}=r_{\text {sh }}$ was assumed. CSH had zero $\mathrm{S}$ and $\mu$, and $\mathrm{r}$ was determined only by the quantum number (k), which depended on Z, but did not depend on the number of pairs.

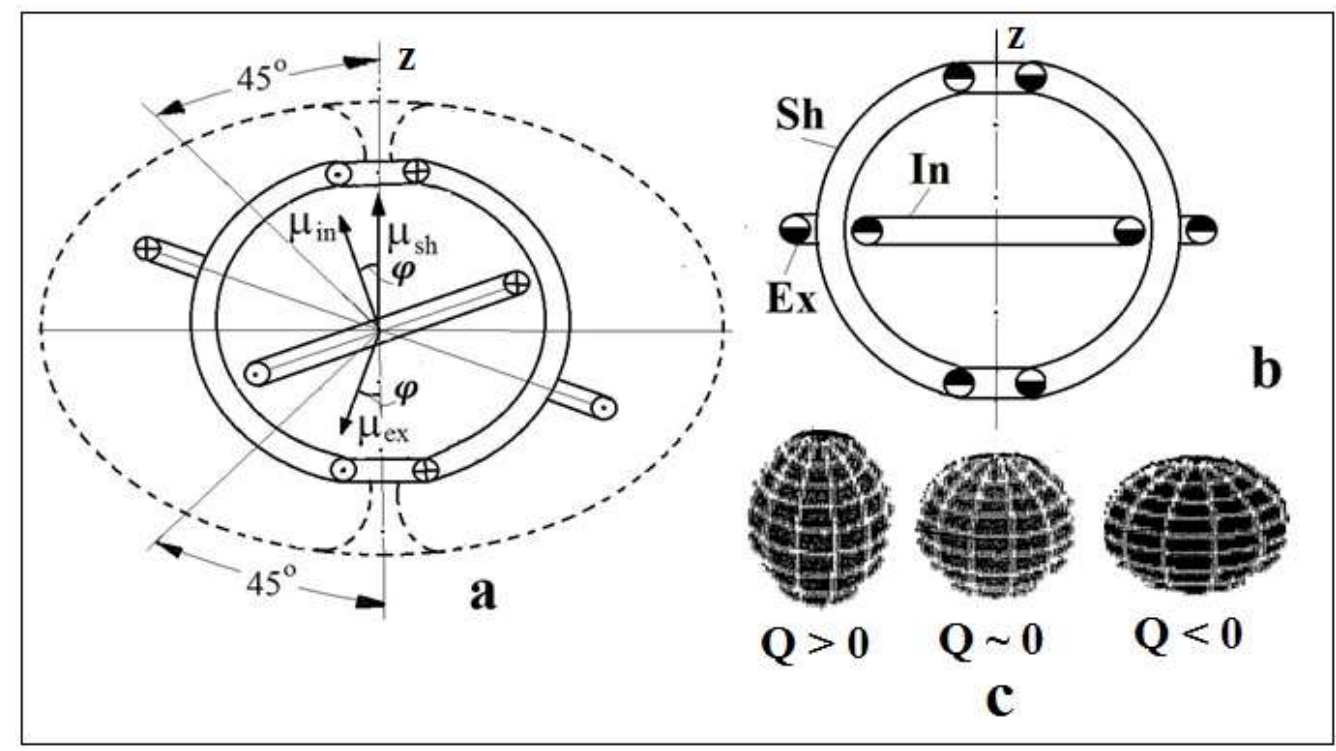


Fig. 2. Schematic diagrams of the device open (a) and closed (b) core shell. Pros, dots and half-filled circles denote the direction of rotation of the elements of the magnetic core of the nucleus. The dashed line designates the region of fluxes of electromagnetic energy corresponding to the nature of the electric charge of the nucleus and the sign of the quadrupole moment $(c)$.

The outer open shells (OSH) of the nucleus (Fig. 2a) had a different number of unpaired neutrons (n) and protons (p). The following types of OSH were calculated: n-like OSH containing n, 3n, 5n; p-like OSH-containing p or 3p; d-like, hybrid OSH of composition: np; $2 n+p, 2 p+n, 3 p+n$. The type of OSH was chosen taking into account the value of $S$ and the sign of $\mathrm{Q}$, while varying the angles of the projections of the vectors $L_{i}$ on the axis of rotation of the nucleus (the $\mathrm{z}$ axis in Fig. 2). The positive sign of $\mathrm{Q}$, that is, the extension of the structure of the nucleus along the $\mathrm{z}$ axis, was related to the deviations of $L_{i}$ by the angles $\varphi=45-90^{\circ}$. The stretching in the direction perpendicular to $\mathrm{z}$ (the negative sign of $\mathrm{Q}$ ) was associated with the deviation of $L_{i}$ by the angles $\varphi=0-45^{\circ}$, (Fig. 2). Correspondingly, the value of $\cos \varphi$ equal to the ratio of the projection $L_{i}$ to the z-axis $\left(L_{i}^{Z}\right)$ to $L_{i}\left(\frac{L_{i}^{Z}}{L_{i}}\right)$ was then less than or greater than $\cos 45$ $\approx 0.76$. When establishing the type of outer shell, the values of the separation energies of the first $\mathrm{n}$ or $\mathrm{p}$ from the nuclei were taken into account, determined by the mass defects (see Table 1). The qualitative consistency between the type of shell and the values of the energies served as an additional criterion in the choice of the nuclear structure model.

Table 1

Типы и энергия отделения нуклонов от внешних оболочек

\begin{tabular}{|c|c|c|c|c|c|c|c|c|c|}
\hline \multirow{3}{*}{$\mathrm{Z}$} & \multirow{3}{*}{ Nucleus } & \multirow{2}{*}{\multicolumn{2}{|c|}{$\begin{array}{c}\text { Energy } \\
\text { separation }(\mathrm{eV})\end{array}$}} & \multirow{3}{*}{$\begin{array}{c}\text { Type of } \\
\text { external } \\
\text { shells }\end{array}$} & \multicolumn{5}{|c|}{ Table continuation } \\
\hline & & & & & 1 & 2 & 3 & 4 & 5 \\
\hline & & n & $\mathbf{P}$ & & 19 & K39 & 13,1 & 6,8 & $3 p$ \\
\hline 1 & 2 & 3 & 4 & 5 & 19 & $\mathrm{~K} 40 *$ & 7,8 & 7,6 & $\mathrm{np}$ \\
\hline 2 & $\mathrm{He} 4$ & 20,6 & 19,8 & $\mathrm{CSH}$ & 20 & $\mathrm{Ca} 40$ & 15,7 & 8,3 & $\mathrm{CSH}$ \\
\hline 3 & Li7 & 7,3 & 10 & $\mathrm{p}$ & 20 & $\mathrm{Ca} 41 *$ & 8,4 & 8,9 & $3 n$ \\
\hline 4 & Be9 & 8,1 & 16,9 & $3 n$ & 21 & Sc45 & 11,4 & 6,9 & $2 n+p$ \\
\hline 5 & B10 & 8,5 & 6,6 & $3 p+n$ & 21 & Sc46* & 8,9 & 8,3 & $\mathrm{np}$ \\
\hline 5 & B11 & 11,4 & 11,2 & $2 n+p$ & 22 & Ti48 & 11,7 & 11,5 & $\mathrm{CSH}$ \\
\hline 5 & $\mathrm{~B} 12 *$ & 3,4 & 14,2 & $\mathrm{np}$ & 22 & Ti49 & 8,2 & 11,6 & $\mathrm{n}$ \\
\hline 6 & $\mathrm{C} 11^{*}$ & 13,1 & 8,8 & $\mathrm{n}$ & 23 & V50* & 9,3 & 7,9 & $3 p+n$ \\
\hline 6 & $\mathrm{C} 12$ & 18,7 & 16 & $\mathrm{CSH}$ & 23 & V51 & 11,1 & 8,1 & $\mathrm{p}$ \\
\hline 6 & $\mathrm{C} 13$ & 5 & 17,6 & $2 p+n$ & 24 & Cr52 & 12,1 & 10,5 & $\mathrm{CSH}$ \\
\hline 6 & C14* & 8,2 & 20,8 & $\mathrm{CSH}$ & 24 & Cr53 & 7,9 & 11,1 & $\mathrm{n}$ \\
\hline 7 & N13* & 20,1 & 1,9 & $2 n+p$ & 25 & Mn52* & 10,6 & 6,6 & $3 p+n$ \\
\hline 7 & N14 & 7,1 & 0,5 & $\mathrm{np}$ & 25 & Mn55 & 10,6 & 8,1 & $3 p$ \\
\hline
\end{tabular}




\begin{tabular}{|c|c|c|c|c|c|c|c|c|c|}
\hline 7 & N15 & 17,9 & 10,2 & $2 n+p$ & 26 & Fe56 & 11,2 & 10,2 & $\mathrm{CSH}$ \\
\hline 8 & O15 & 13,2 & 14,3 & $\mathrm{n}$ & 26 & Fe57 & 7,7 & 10,6 & $\mathrm{n}$ \\
\hline 8 & O16 & 15,6 & 15,6 & $\mathrm{CSH}$ & 27 & Co59 & 10,5 & 7,3 & $3 p$ \\
\hline 8 & O17 & 4,2 & 13,9 & $3 n$ & 28 & Ni58 & 12,2 & 8,2 & $\mathrm{CSH}$ \\
\hline 9 & F17* & 16,8 & 0,6 & $3 p$ & 28 & Ni61 & 7,8 & 9,9 & $\mathrm{n}$ \\
\hline 9 & F19 & 10,4 & 8,0 & $\mathrm{p}$ & 29 & Cu63 & 10,7 & 6,3 & $2 n+p$ \\
\hline 10 & $\mathrm{Ne} 20$ & 16,9 & 12,8 & $\mathrm{CSH}$ & 30 & Zn64 & 11,9 & 7,7 & $\mathrm{CSH}$ \\
\hline 10 & $\mathrm{Ne} 21$ & 6,8 & 12,8 & $\mathrm{n}$ & 30 & Zn65* & 8,0 & 7,7 & $3 n$ \\
\hline 11 & $\mathrm{Na} 23$ & 12,4 & 8,8 & $3 p$ & 31 & Ga68* & 8,3 & 6,5 & $\mathrm{np}$ \\
\hline 12 & Mg24 & 16,5 & 11,8 & $\mathrm{CSH}$ & 31 & Ga69 & 10,3 & 9 & $\mathrm{p}$ \\
\hline 12 & Mg25 & 8,8 & 12,1 & $\mathrm{n}$ & 32 & Ge70 & 11,6 & 8,6 & $\mathrm{CSH}$ \\
\hline 13 & $\mathrm{Al} 27$ & 21,3 & 8,3 & $3 p$ & 32 & Ge71* & 7,4 & 8,3 & $\mathrm{n}$ \\
\hline 13 & $\mathrm{Al} 28 *$ & 7,7 & 9,5 & $\mathrm{np}$ & 32 & Ge73* & 6,7 & 10 & $5 n$ \\
\hline 14 & Si28 & 17,2 & 11,6 & $\mathrm{CSH}$ & 33 & As75 & 10,2 & 6,9 & $3 p$ \\
\hline 14 & $\mathrm{Si} 29$ & 8,5 & 12,4 & $\mathrm{n}$ & 34 & Se77 & 6,4 & 9,6 & $\mathrm{n}$ \\
\hline 15 & P31 & 12,3 & 7,3 & $\mathrm{p}$ & 34 & Se80 & 9,9 & 11,3 & $\mathrm{CSH}$ \\
\hline 16 & S32 & 11,2 & 18,2 & $\mathrm{CSH}$ & 35 & Br81 & 10,2 & 7,1 & $3 p$ \\
\hline 16 & S33 & 8,7 & 9,6 & $3 n$ & 35 & $\mathrm{Br} 82 *$ & 7,6 & 8,4 & $3 p+n$ \\
\hline 17 & $\mathrm{Cl} 35$ & 12,7 & 6,4 & $2 n+p$ & 36 & Kr83 & 7,5 & 9,8 & $3 n$ \\
\hline 17 & $\mathrm{Cl} 36^{*}$ & 8,6 & 8,0 & $3 n+p$ & 36 & Kr84 & 10,5 & 10,7 & $\mathrm{CSH}$ \\
\hline 18 & Ar36 & 15,6 & 8,3 & $\mathrm{CSH}$ & 37 & $\mathrm{Rb85}$ & 10,5 & 7,1 & $3 p$ \\
\hline 18 & Ar40 & 9,9 & 12,5 & $\mathrm{CSH}$ & 37 & $\mathrm{Rb} 86$ & 8,6 & 8,5 & np \\
\hline
\end{tabular}

The parameters of the CSH and OSH structure were calculated by solving the systems of equations (1) - (5) taking into account conditions (6) - (9). The empirical value of the magnetic moment of the nucleus $\mu_{f}=f \mu_{N}$ ( $\mu_{N}$ is the Bohr nuclear magneton) was equated to the sum of the projections $\mu_{i}=\frac{L_{i} \cos \varphi\left|q_{i}\right|}{2 m_{i} C} \mathrm{Sh}$, In and Ex of the outer OSH. For an OSH containing $\mathrm{j}$ nucleons, the mass $\mathrm{m}=j m_{N}$, где $m_{N}=m_{n} \cong m_{p}=1,6710^{-24}$ г.

$L_{s h}=\frac{2}{3} m_{s h} r^{2}{ }_{s h} w_{s h}=\frac{2 k-1}{2} \hbar,(\mathbf{1}) ;$

$L_{i n}=m_{i n} r_{s h}^{2} w_{s h}=k \hbar,(2)$;

$L_{e x}=m_{e x} r_{e x}^{2} w_{e x}=-k \hbar,(\mathbf{3}) ;(\mathbf{1})-(\mathbf{3})-$ quantization of rotational moments.

$\mu_{f}=f \mu_{N}=f j \frac{e \hbar}{2 m C}=\sum \frac{L_{i}\left|q_{i}\right|}{2 m_{i} C},(4)$ - expression for the magnetic moment of the nucleus.

$\frac{3}{2} \sum L_{i} w_{i}=m C^{2},(\mathbf{5})$ - equality of rotational energy and rest energy of the nucleus.

$w_{i} r_{i}=$ const, $(\mathbf{6})-$ condition of wholeness of the vortex structure of the nucleus.

$S=\sum L_{i},(\mathbf{7}) ; \mathrm{m}=\sum m_{i}(\mathbf{8}) ; q_{s h}=q_{\text {in }}=\frac{2}{3} e ; q_{e x}=-\frac{1}{3} e,(\mathbf{9})$. 
The index $\mathrm{i}$ in the sums and in (6) corresponds to Sh, In, Ex; e is the unit charge. The condition (9) for p: $q_{s h}=q_{\mathrm{ex}}=\frac{2}{3} e ; q_{i n}=-\frac{1}{3} e ;$ для $\mathbf{n}: q_{s h}=\frac{2}{3} e ; q_{e x}=-\frac{2}{3} e$. We note that in the structure of the In and Ex nuclei, the orbitals of p are interchanged. Equations (1) - (3), (5) - (7) for $\mathrm{CSH}$ of the nucleus $\mathrm{He}^{4}$ were computed by representing $\mathrm{CSH}$ consisting of two correlated pairs (pp) and (nn) c k =2. The solution of the system for helium-like CSH, with any number of quadruples $[(\mathrm{nn})(\mathrm{pp})]$ gives a recurrence formula for the $\mathrm{CSH}$ radius.

$$
r_{30}=\frac{3 \hbar}{4 m_{N} C}[(4 k-1)(5 k-1)]^{0,5}=0,158[(4 k-1)(5 k-1)]^{0,5} \mathrm{Fm} .
$$

The condensation of two quadruples $[(\mathrm{nn})(\mathrm{pp})]$ forms the unstable structure of the doubly "magic" isotope $\mathrm{Be} 8^{*}$, which decays after $\sim 10^{-17} \mathrm{~s}$ into two $\alpha$-particles. Taking this into account, formula (10) was used except for $\mathrm{He}^{4}$ only for $\mathrm{Ne} 20$ and $\mathrm{Ar} 36$, while for other nuclei external CSHs were composed of isolated CSH (nn)-type and (pp)-type. The number of pairs in these shells could differ. The recurrence formulas for the radii and mass ratios of the elements of these CSHs were:

$$
\begin{aligned}
& r_{n n}=\frac{\hbar}{4 m_{N} C}[3(4 k-1)(10 k-3)]^{0,5}=0,091[(4 k-1)(10 k-3)]^{0,5} \mathrm{Fm}, \\
& \frac{m_{s h}}{m} 100=\frac{3(2 k-1)}{10 k-3} \% ; \frac{m_{e x}}{m} 100=\frac{4 k}{10 k-3} \%, \\
& r_{p p}=\frac{\hbar}{4 m_{N} C}[3(6 k-1)(14 k-3)]^{0,5}=0,091[(6 k-1)(14 k-3)]^{0,5} \mathrm{Fm}, \\
& \frac{m_{s h}}{m} 100=\frac{3(2 k-1)}{14 k-3} \% ; \quad \frac{m_{\text {in }}}{m} 100=\frac{4 k}{14 k-3} \% ; m_{\text {in }}=m_{\text {ex }},
\end{aligned}
$$

It is known [10] that $\mathrm{r}_{-} \mathrm{e}$ differed not more than $1-2 \%$ in Ca isotopes and transition metals, and r_e of $\mathrm{Zn}$ isotopes was larger than r_e of Ca isotopes by $~ 12 \%$. Taking these data into account, the selection of $\mathrm{k}$ in formulas (11) - (14) took into account the results of calculations of isotopes with OSH and fixed the values of $\mathrm{k}$ in a series of related elements. For example, for nuclei with an external CSH (pp) type, $\mathrm{k}_{\mathrm{pp}}=5$ for the $\mathrm{Mg} 24-\mathrm{Ar} 40$ and $\mathrm{k}_{\mathrm{pp}}=6$ series for the Ca40-Se80 series were taken in the formula (13). In this case, the number $\mathrm{k}_{\mathrm{nn}}$ in formula (11) increased as the number of (nn) pairs in CSH increased and, beginning with Ge70, $\mathrm{k}_{\mathrm{nn}}=9$ was taken. In these cases, the shell of the (nn) -type became external.

For example, we show the scheme for calculating the OSH $(2 n+p)$-type for the nucleus $\mathrm{Cl} 35\left(\mathrm{~S}=1,5 \hbar, \mu=0,82 \mu_{N} ; \mathrm{Q}=-1,8 \mathrm{Fm}^{2}\right)$. For $\mathrm{k}=6$ we have $L_{\text {in }}=6 \hbar ; L_{s h}=16,5 \hbar ; L_{e x}=$ $-18 \hbar$. To obtain $S=1.5 \hbar$ and negative sign $\mathrm{Q}$, the vector $L_{s h}$ must be rotated by an angle $\varphi$ at which $\cos \varphi=\frac{L_{s h}^{Z}}{L_{s h}}=\frac{13,5}{16,5}(13,5 / 16,5=0,818>0,76)$. Under these conditions, equations (1) (5) have the form: 


$$
\begin{aligned}
& \frac{2}{3} \frac{m_{s h}}{3} r^{2}{ }_{s h} w_{s h}=\frac{11}{2} \hbar ; m_{i n} r^{2}{ }_{s h} w_{s h}=6 \hbar ; \frac{m_{e x}}{3} r^{2}{ }_{e x} w_{e x}=-6 \hbar ; \\
& 0,82 \frac{3 e \hbar}{2 m C}=\frac{13,5}{3} \hbar \frac{2}{3} e \frac{3}{m_{s h}} 3+6 \hbar \frac{2}{3} e \frac{1}{m_{\text {in }}}-6 \hbar\left(\frac{1}{3}+\frac{4}{3}\right) e \frac{3}{m_{e x}} \\
& \frac{3}{2} \hbar\left(16,5 w_{s h}+6 w_{i n}+18 w_{e x}\right)=m C^{2} .
\end{aligned}
$$

Solving these equations, taking into account conditions (6) - (9) for $\mathrm{m}=3 \mathrm{~m} \_\mathrm{N}$, we obtained the $\mathrm{CSH}$ parameters for $\mathrm{Cl} 35$ (see Table 2). When calculating parameters, the tabular values were rounded to the second decimal point and the kernel parameters were calculated with the same accuracy.

\section{RESULTS AND DISCUSSION}

The results of calculations of 129 stable and unstable isotopes of elements with $\mathrm{Z}$ from 1 to 37 are given in Table 2. In this case, the total number of variants of structural calculations exceeded 260, that is, on average, each calculation was duplicated and a variant was chosen that best suited the experimental data.

The dependence of $r_{e x}$ or $r_{s h}$ (in the case $r_{e x}<r_{s h}$ ) on $\mathrm{Z}$ is in good agreement with the empirical dependence $\llbracket r=r_{0} A^{1 / 3}$ for $1,2<\mathrm{r}_{0}<1,5$ [5] (Fig. 3). The calculated radii were compared with the known dimensions of the nuclei obtained in experiments on scattering by nuclei of different particles and by laser spectroscopy of atoms. These methods allow us to calculate $r_{e}$. For example, the first method for He3, He4, Be9, C12 nuclei yields r_e: 1.97; 1.68; 2.52; $2.37 \mathrm{Fm}[10,11]$. For unstable isotopes, $\mathrm{He}^{*}, \mathrm{He} 8^{*} ; \mathrm{Be} 7^{*}, \mathrm{Be} 10^{*}, \mathrm{Be} 11^{*}$ the second method gives $r_{e}: 2.07 ; 1.93 ; 2.65 ; 2.35 ; 2.45 \mathrm{Fm}[12.13]$, respectively. Since $r_{e}$ characterizes the Kelvin oval [14] (Fig. 2), $r_{e x}$ and $r_{s h}$ can be smaller than $r_{e}$, especially for OSH.

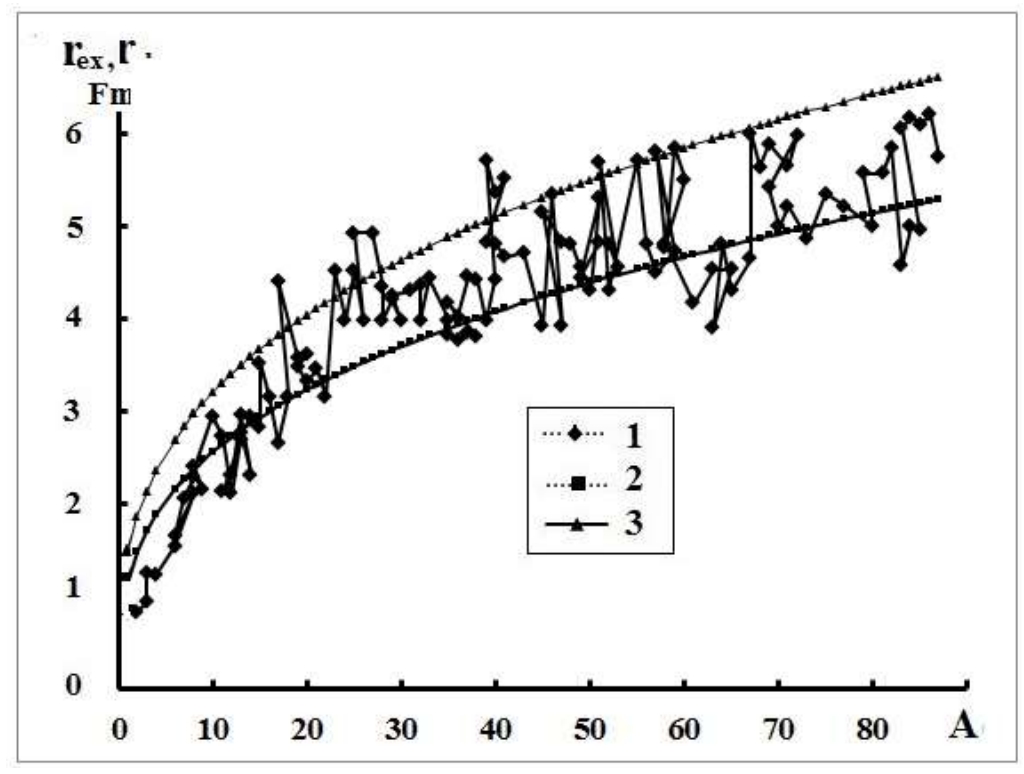

Fig. 3. Dependence on the mass number A of the radii of the vortex structure of the nucleus (1) and the empirical dependence $r_{0} A^{1 / 3} \mathrm{c} r_{0}=1,2$ (2) and 1,5 (3). 
Table 2

Structure and parameters of the outer shells of nuclei

\begin{tabular}{|c|c|c|c|c|c|c|c|c|c|}
\hline \multirow{3}{*}{$\begin{array}{l}\mathrm{Z} \\
0\end{array}$} & \multirow{3}{*}{$\begin{array}{l}\text { Nucleus } \\
\mathrm{n}\end{array}$} & \multirow{3}{*}{$\begin{array}{l}\text { Structure } \\
\text { Sh-Ex }\end{array}$} & \multirow{3}{*}{$\begin{array}{c}w \\
10^{-22} \\
\left(c^{-1}\right) \\
120\end{array}$} & $m_{s h}$ & $m_{e x}$ & \multirow{3}{*}{$L_{i} \cos \varphi$} & $r_{s h}\left(r_{n n}\right)$ & $r_{e x}\left(r_{p p}\right)$ & \multirow{3}{*}{$\begin{array}{c}k^{(1} \\
\\
1\end{array}$} \\
\hline & & & & \multicolumn{2}{|c|}{$\%$} & & \multicolumn{2}{|c|}{$\mathrm{Fm}$} & \\
\hline & & & & 72 & 28 & & 0,24 & 0,85 & \\
\hline 1 & $\mathrm{p}$ & In-Sh-Ex & 45 & 32 & 26 & & 0,58 & 0,93 & 1 \\
\hline 1 & d & (pn) & 64 & 43 & 28 & Ex0,5/1 & 0,41 & 0,85 & 1 \\
\hline 1 & $\mathrm{t}$ & $(\mathrm{nn}) \mathrm{p}$ & 81 & 53 & 23 & & 0,33 & 0,96 & 1 \\
\hline 2 & $\mathrm{He} 3$ & (pp)n & 75 & 41 & 23 & & 0,8 & 1,27 & 2 \\
\hline 2 & $\mathrm{He} 4$ & $(\mathrm{nn})(\mathrm{pp})$ & 21 & 43 & 38 & & 1,25 & 1,25 & 2 \\
\hline 2 & $\mathrm{He}^{*}$ & $2(\mathrm{nn})(\mathrm{pp})$ & 17 & 56 & 44 & & 1,57 & 1,51 & $3-2$ \\
\hline 2 & $\mathrm{He} 8 *$ & $3(\mathrm{nn})(\mathrm{pp})$ & 13 & 57 & 43 & & 2,14 & 1,51 & $4-2$ \\
\hline 3 & Li6 & $(\mathrm{pp}) 3 \mathrm{n}+\mathrm{p}$ & 25 & 50 & 40 & Ex 7/8 & 1,52 & 1,68 & 2 \\
\hline 3 & Li7 & $2(\mathrm{nn})(\mathrm{pp}) \mathrm{p}$ & 20 & 41 & 23 & & 1,32 & 2,09 & 2 \\
\hline 3 & Li 8* & 2(nn)(pp)np & 15 & 50 & 30 & & 1,76 & 2,32 & 3 \\
\hline 4 & $\mathrm{Be} 9$ & $(\mathrm{nn}) 2(\mathrm{pp}) 3 \mathrm{n}$ & 12 & 60 & 40 & Ex9/12 & 2,17 & 2,1 & 4 \\
\hline 5 & B8* & (nn)2(pp)np & 16 & 51 & 29 & & 1,74 & 2,42 & 3 \\
\hline 5 & B10 & $2(n n)(p p) 3 p+n$ & 14 & 48 & 24 & In $5 / 9$ & 1,85 & 2,97 & 3 \\
\hline 5 & B11 & $2(n n) 2(p p) 2 n+p$ & 13 & 43 & 39 & In $3 / 4$ & 1,89 & 2,75 & 4 \\
\hline 5 & B12* & 3(nn)2(pp)np & 14 & 48 & 33 & In $2 / 3$ & 1,86 & 2,13 & 3 \\
\hline 5 & B13* & $3(\mathrm{nn}) 2(\mathrm{pp}) 2 \mathrm{n}+\mathrm{p}$ & 12 & 42 & 39 & In $3 / 4$ & 1,9 & 2,72 & 4 \\
\hline 6 & C11* & 2(nn)3(pp)n & 13 & 57 & 43 & Ex 2/4 & 2,13 & 2,15 & 4 \\
\hline 6 & $\mathrm{C} 12$ & $3(\mathrm{nn}) 3(\mathrm{pp})$ & 11 & 38 & 31 & & 1,57 & 2,34 & 3 \\
\hline 6 & $\mathrm{C} 13$ & $3(\mathrm{nn}) 2(\mathrm{pp}) 2 \mathrm{p}+\mathrm{n}$ & 14 & 46 & 30 & In $2 / 6$ & 2,36 & 2,98 & 3 \\
\hline 6 & C14* & $4(\mathrm{nn}) 3(\mathrm{pp})$ & 11 & 38 & 31 & & 2,14 & 2,34 & $4-3$ \\
\hline 7 & N13* & $2(n n) 3(p p) 2 n+p$ & 13 & 44 & 38 & In $2 / 4$ & 1,85 & 2,8 & 4 \\
\hline 7 & N14 & 3(nn)3(pp)np & 11 & 49 & 32 & In $2 / 4$ & 2,5 & 2,96 & 4 \\
\hline 7 & N15 & $3(n n) 3(p p) 2 n+p$ & 13 & 44 & 37 & In $2 / 4$ & 1,82 & 2,86 & 4 \\
\hline 8 & O15 & 3(nn)4(pp)n & 11 & 45 & 55 & Ex 4/5 & 3,55 & 2,15 & 5 \\
\hline 8 & O16 & 4(nn)4(pp) & 8,3 & 40 & 30 & & 2,14 & 3,18 & 4 \\
\hline 8 & O17 & $3(\mathrm{nn}) 4(\mathrm{pp}) 3 \mathrm{n}$ & 10 & 58 & 42 & Ex11/15 & 2,62 & 2,68 & 5 \\
\hline 8 & $\mathrm{O} 18$ & $5(\mathrm{nn}) 4(\mathrm{pp})$ & 8,3 & 40 & 30 & & 2,72 & 3,18 & $5-4$ \\
\hline 9 & $\mathrm{~F} 17^{*}$ & $4(n n) 3(p p) 3 p$ & 9,5 & 45 & 21 & In $4 / 12$ & 2,79 & 4,43 & 4 \\
\hline 9 & F19 & 5(nn)4(pp)p & 8,8 & 42 & 32 & In $1 / 4$ & 3 & 3,61 & 4 \\
\hline 9 & F20* & 5(nn)4(pp)np & 8,4 & 50 & 32 & In $3 / 5$ & 3,21 & 3,64 & 5 \\
\hline 10 & Ne19* & $4(\mathrm{nn}) 5(\mathrm{pp}) \mathrm{n}$ & 7,5 & 61 & 39 & Ex4/5 & 3,05 & 3,51 & 5 \\
\hline 10 & $\mathrm{Ne} 20$ & $5(\mathrm{nn}) 5(\mathrm{pp})$ & 7,9 & 47 & 35 & & 3,37 & 3,37 & 5 \\
\hline 10 & $\mathrm{Ne} 21$ & $5(\mathrm{nn}) 5(\mathrm{pp}) \mathrm{n}$ & 8,7 & 60 & 40 & Ex4/6 & 3,14 & 3,48 & 6 \\
\hline 10 & $\mathrm{Ne} 22$ & $6(\mathrm{nn}) 5(\mathrm{pp})$ & 8,3 & 40 & 30 & 9,2 & 2,72 & 3,18 & $5-4$ \\
\hline 11 & $\mathrm{Na} 23$ & $6(n n) 4(p p) 3 p$ & 9,6 & 45 & 21 & In $3 / 15$ & 2,77 & 4,54 & 4 \\
\hline 12 & Mg24 & $6(\mathrm{nn}) 6(\mathrm{pp})$ & 6,6 & 40 & 30 & & 2,72 & 4,01 & 5 \\
\hline
\end{tabular}




\begin{tabular}{|c|c|c|c|c|c|c|c|c|c|}
\hline 12 & $\mathrm{Mg} 25$ & 6(nn)6(pp)n & 8,1 & 65 & 35 & Ex4/7 & 3,41 & 4,55 & 7 \\
\hline 12 & Mg26 & $7(\mathrm{nn}) 6(\mathrm{pp})$ & 6,6 & 40 & 30 & & 3,29 & 4,01 & $6-5$ \\
\hline 13 & $\mathrm{~A} 125^{*}$ & $6(n n) 5(p p) 3 p$ & 7,8 & 45 & 21 & In $4 / 12$ & 3,09 & 4,95 & 4 \\
\hline 13 & Al27 & $7(n n) 5(p p) 3 p$ & 7,8 & 45 & 21 & In $4 / 12$ & 3,09 & 4,95 & 4 \\
\hline 13 & $\mathrm{Al} 28 *$ & 7(nn)6(pp)np & 6,9 & 50 & 32 & In $4 / 6$ & 3,9 & 4,38 & 6 \\
\hline 14 & Si28 & 7(nn)7(pp) & 6,6 & 40 & 30 & & 2,72 & 4,01 & 5 \\
\hline 14 & Si29 & 7(nn)7(pp)n & 6,4 & 54 & 46 & Ex6/7 & 4,23 & 3,53 & 7 \\
\hline 14 & Si30 & $8(\mathrm{nn}) 7(\mathrm{pp})$ & 6,6 & 40 & 30 & & 3,87 & 4,01 & $7-5$ \\
\hline 15 & P29* & 7(nn)7(pp)p & 9,4 & 44 & 22 & In $1 / 4$ & 2,82 & 4,28 & 4 \\
\hline 15 & P31 & 8(nn)7(pp)p & 9,4 & 44 & 22 & In $1 / 4$ & 2,81 & 4,33 & 5 \\
\hline 15 & P32* & 8(nn)7(pp)np & 6,9 & 50 & 32 & In $2 / 6$ & 3,9 & 4,39 & 6 \\
\hline 16 & S32 & $8(\mathrm{nn}) 8(\mathrm{pp})$ & 6,6 & 40 & 30 & & 3,87 & 4,01 & $7-5$ \\
\hline 16 & S33 & $7(\mathrm{nn}) 8(\mathrm{pp}) 3 \mathrm{n}$ & 6,8 & 51 & 49 & Ex18/21 & 4,46 & 3,35 & 7 \\
\hline 16 & S35* & $8(\mathrm{nn}) 8(\mathrm{pp}) 3 \mathrm{n}$ & 6,8 & 56 & 44 & Ex4/7 & 4,01 & 3,71 & 7 \\
\hline 17 & $\mathrm{Cl} 35$ & $8(\mathrm{nn}) 8(\mathrm{pp}) 2 \mathrm{n}+\mathrm{p}$ & 7,1 & 51 & 36 & Sh13,5/16,5 & 3,8 & 3,85 & 6 \\
\hline 17 & $\mathrm{Cl}^{2} 6^{*}$ & $8(n n) 8(p p) 3 n+p$ & 7,1 & 51 & 40 & Sh20/24 & 3,8 & 3,55 & 6 \\
\hline 17 & $\mathrm{Cl} 37$ & $9(\mathrm{nn}) 8(\mathrm{pp}) 2 \mathrm{n}+\mathrm{p}$ & 7,1 & 52 & 36 & Sh13,5/16,5 & 3,79 & 3,87 & 6 \\
\hline 17 & $\mathrm{Cl} 38$ & $9(\mathrm{nn}) 8(\mathrm{pp}) 3 \mathrm{n}+\mathrm{p}$ & 7 & 51 & 40 & Sh20/24 & 3,84 & 3,51 & 6 \\
\hline 18 & Ar35 & $7(\mathrm{nn}) 9(\mathrm{pp}) 3 \mathrm{n}$ & 6,4 & 54 & 46 & Ex18/21 & 4,19 & 3,55 & 7 \\
\hline 18 & Ar36 & $9(\mathrm{nn}) 9(\mathrm{pp})$ & 6,6 & 48 & 35 & & 4,08 & 4,08 & 6 \\
\hline 18 & Ar37 & $8(\mathrm{nn}) 9(\mathrm{pp}) 3 \mathrm{n}$ & 6,8 & 51 & 49 & Ex18/21 & 4,48 & 3,34 & 7 \\
\hline 18 & Ar38 & $10(\mathrm{nn}) 9(\mathrm{pp})$ & 5,8 & 58 & 41 & 0,06 & 4,45 & 4,01 & $8-5$ \\
\hline 18 & Ar39 & $9(\mathrm{nn}) 9(\mathrm{pp}) 3 \mathrm{n}$ & 6,8 & 57 & 43 & Ex16/21 & 4 & 3,72 & 7 \\
\hline 18 & Ar40 & 11(nn)9(pp) & 5,8 & 58 & 41 & & 4,45 & 4,01 & $8-5$ \\
\hline 19 & K39 & $10(\mathrm{nn}) 8(\mathrm{pp}) 3 \mathrm{p}$ & 7,6 & 46 & 21 & In3/15 & 3,5 & 5,74 & 5 \\
\hline 19 & $\mathrm{~K} 40^{*}$ & 10(nn)9(pp)np & 7,6 & 54 & 26 & In5/6 & 3,56 & 5,39 & 6 \\
\hline 19 & K41 & $11(\mathrm{nn}) 8(\mathrm{pp}) 3 \mathrm{p}$ & 7,4 & 50 & 20 & In3/15 & 3,78 & 5,55 & 5 \\
\hline 20 & Ca39* & $9(\mathrm{nn}) 10(\mathrm{pp}) \mathrm{n}$ & 5,7 & 53 & 47 & Ex5/7 & 4,86 & 3,86 & 8 \\
\hline 20 & $\mathrm{Ca} 40$ & $10(\mathrm{nn}) 10(\mathrm{pp})$ & 5,4 & 40 & 30 & & 4,45 & 4,84 & $8-6$ \\
\hline 20 & $\mathrm{Ca} 41 *$ & $9(\mathrm{nn}) 10(\mathrm{pp}) 3 \mathrm{n}$ & 5,8 & 56 & 44 & Ex19/24 & 4,69 & 4,19 & 8 \\
\hline 20 & $\mathrm{Ca} 43$ & $10(\mathrm{nn}) 10(\mathrm{pp}) 3 \mathrm{n}$ & 5,7 & 55 & 45 & Ex19/24 & 4,74 & 3,38 & 8 \\
\hline 21 & Sc45 & $11(\mathrm{nn}) 10(\mathrm{pp}) 2 \mathrm{n}+\mathrm{p}$ & 6,7 & 50 & 38 & In $5 / 6$ & 3,95 & 3,74 & 6 \\
\hline 21 & Sc46* & 12(nn)10(pp)np & 6 & 51 & 31 & In $5 / 7$ & 4,45 & 5,38 & 7 \\
\hline 21 & Sc47* & $12(\mathrm{nn}) 10(\mathrm{pp}) 2 \mathrm{n}+\mathrm{p}$ & 6,8 & 50 & 38 & In $5 / 6$ & 3,94 & 3,68 & 6 \\
\hline 22 & Ti45* & 11(nn)11(pp)n & 7 & 65 & 35 & Ex4/8 & 3,95 & 5,19 & 8 \\
\hline 22 & Ti47 & 12(nn)11(pp)n & 6,6 & 62 & 38 & Ex5/8 & 4,13 & 4,86 & 8 \\
\hline 22 & Ti48 & 13(nn)11(pp) & 5,4 & 40 & 30 & & 4,45 & 4,84 & $8-6$ \\
\hline 22 & Ti49 & 13(nn)11(pp)n & 8,2 & 65 & 35 & Ex4/7 & 3,39 & 4,58 & 7 \\
\hline 23 & V50* & $13(\mathrm{nn}) 10(\mathrm{pp}) 3 \mathrm{p}+\mathrm{n}$ & 7 & 42 & 34 & In $8 / 15$ & 4,34 & 4,1 & 5 \\
\hline 23 & V51 & 14(nn)11(pp)p & 7,4 & 45 & 22 & In $4 / 5$ & 3,58 & 5,34 & 5 \\
\hline 24 & $\mathrm{Cr} 49^{*}$ & 12(nn)12(pp)n & 6,2 & 59 & 41 & Ex5/8 & 4,42 & 4,47 & 8 \\
\hline 24 & Cr51* & 13(nn)12(pp)n & 6,7 & 62 & 38 & Ex4/8 & 4,13 & 4,86 & 8 \\
\hline 24 & Cr52 & 14(nn)12(pp) & 5,4 & 40 & 30 & & 4,45 & 4,84 & $8-6$ \\
\hline
\end{tabular}




\begin{tabular}{|c|c|c|c|c|c|c|c|c|c|}
\hline 24 & Cr53 & 14(nn)12(pp)n & 5,9 & 57 & 43 & Ex6/8 & 4,58 & 4,28 & 8 \\
\hline 25 & Mn51* & 13(nn)11(pp)3p & 7,6 & 45 & 21 & In $4 / 15$ & 3,51 & 5,72 & 5 \\
\hline 25 & $\operatorname{Mn} 52^{*}$ & $13(n n) 11(p p) 3 p+n$ & 7 & 43 & 33 & In $8 / 15$ & 4,34 & 4,14 & 5 \\
\hline 25 & Mn55 & $15(\mathrm{nn}) 11(\mathrm{pp}) 3 \mathrm{p}$ & 7,6 & 45 & 21 & In $4 / 15$ & 3,5 & 5,74 & 5 \\
\hline 26 & $\mathrm{Fe} 56$ & 15(nn)13(pp) & 5,4 & 40 & 30 & & 4,45 & 4,84 & $8-6$ \\
\hline 26 & $\mathrm{Fe} 57$ & $15(\mathrm{nn}) 13(\mathrm{pp}) \mathrm{n}$ & 5,9 & 50 & 50 & Ex6/7 & 4,52 & 3,3 & 7 \\
\hline 26 & $\mathrm{Fe} 59 *$ & $16(\mathrm{nn}) 13(\mathrm{pp}) \mathrm{n}$ & 5,7 & 55 & 45 & Ex6/8 & 4,76 & 4,14 & 8 \\
\hline 27 & Co57* & $15(\mathrm{nn}) 12(\mathrm{pp}) 3 \mathrm{p}$ & 7,6 & 46 & 20 & In $5 / 15$ & 3,49 & 5,83 & 5 \\
\hline 27 & Co58* & 15(nn)13(pp)np & 5,6 & 48 & 35 & In $3 / 7$ & 4,8 & 4,7 & 7 \\
\hline 27 & Co59 & $16(\mathrm{nn}) 12(\mathrm{pp}) 3 \mathrm{p}$ & 7,6 & 46 & 20 & In $5 / 15$ & 3,48 & 5,88 & 5 \\
\hline 27 & Co60* & $16(n n) 12(p p) 3 p+n$ & 8,2 & 48 & 25 & In $7 / 15$ & 3,79 & 5,53 & 5 \\
\hline 28 & $\mathrm{Ni58}$ & 15(nn)14(pp) & 5,4 & 40 & 30 & & 4,45 & 4,84 & $8-6$ \\
\hline 28 & Ni61 & $16(\mathrm{nn}) 14(\mathrm{pp}) \mathrm{n}$ & 8,8 & 61 & 39 & Ex4/6 & 3,73 & 4,19 & 6 \\
\hline 29 & Cu63 & $16(n n) 14(p p) 2 n+p$ & 5,9 & 50 & 38 & Sh15,5/19,5 & 4,56 & 4,38 & 7 \\
\hline 29 & $\mathrm{Cu} 65$ & $17(n n) 14(p p) 2 n+p$ & 5,9 & 50 & 38 & Sh15,5/19,5 & 4,56 & 4,38 & 7 \\
\hline 30 & Zn63* & $16(\mathrm{nn}) 15(\mathrm{pp}) \mathrm{n}$ & 6,9 & 57 & 43 & Ex5/7 & 3,93 & 3,81 & 7 \\
\hline 30 & Zn64 & $17(\mathrm{nn}) 15(\mathrm{pp})$ & 5,4 & 40 & 30 & & 4,45 & 4,84 & $8-6$ \\
\hline 30 & Zn65* & $16(\mathrm{nn}) 15(\mathrm{pp}) 3 \mathrm{n}$ & 6,2 & 53 & 47 & Ex17/21 & 4,33 & 3,45 & 7 \\
\hline 30 & Zn67 & $18(\mathrm{nn}) 15(\mathrm{pp}) \mathrm{n}$ & 7,1 & 59 & 41 & Ex4/7 & 4,57 & 4,68 & 7 \\
\hline 31 & Ga67* & $18(\mathrm{nn}) 15(\mathrm{pp}) \mathrm{p}$ & 7,7 & 46 & 20 & In $2 / 5$ & 3,46 & 6,03 & 5 \\
\hline 31 & Ga68* & 18(nn)15(pp)np & 5 & 49 & 33 & In $2 / 8$ & 5,31 & 5,67 & 8 \\
\hline 31 & Ga69 & $19(\mathrm{nn}) 15(\mathrm{pp}) \mathrm{p}$ & 7,6 & 46 & 20 & In $2 / 5$ & 3,47 & 5,92 & 5 \\
\hline 31 & Ga71 & 20(nn)15(pp)p & 7,5 & 45,5 & 21 & In $2 / 5$ & 3,52 & 5,68 & 5 \\
\hline 31 & Ga72* & 20(nn)15(pp)np & 4,8 & 50 & 32 & In $4 / 8$ & 5,44 & 6,01 & 8 \\
\hline 32 & Ge69* & $18(\mathrm{nn}) 16(\mathrm{pp}) \mathrm{n}$ & 4,9 & 48 & 52 & Ex5/8 & 5,45 & 3,66 & 8 \\
\hline 32 & Ge70 & 19(nn)16(pp) & 5,4 & 58 & 42 & & 5,02 & 4,84 & $9-6$ \\
\hline 32 & Ge71* & $19(\mathrm{nn}) 16(\mathrm{pp}) \mathrm{n}$ & 5,1 & 50 & 50 & Ex5/8 & 5,24 & 3,78 & 8 \\
\hline 32 & Ge73* & $18(\mathrm{nn}) 16(\mathrm{pp}) 5 \mathrm{n}$ & 5,5 & 54 & 46 & Ex33/40 & 4,89 & 4 & 8 \\
\hline 33 & As75 & $21(\mathrm{nn}) 15(\mathrm{pp}) 3 \mathrm{p}$ & 7,4 & 45 & 22 & In $3 / 15$ & 3,57 & 5,38 & 5 \\
\hline 34 & $\mathrm{Se} 77$ & 21(nn)17(pp)n & 5,1 & 50 & 50 & Ex7/8 & 5,24 & 3,77 & 8 \\
\hline 34 & Se80 & 23(nn)17(pp) & 5,4 & 58 & 42 & & 5,02 & 4,84 & $9-6$ \\
\hline 35 & Br79 & $22(\mathrm{nn}) 16(\mathrm{pp}) 3 \mathrm{p}$ & 7,5 & 45 & 21 & In $3 / 15$ & 3,52 & 5,6 & 5 \\
\hline 35 & Br81 & $23(\mathrm{nn}) 16(\mathrm{pp}) 3 \mathrm{p}$ & 7,5 & 45 & 21 & In $3 / 15$ & 3,52 & 5,6 & 5 \\
\hline 35 & $\mathrm{Br} 82 *$ & $23(n n) 16(p p) 3 p+n$ & 8,1 & 49 & 24 & In $7 / 15$ & 3,76 & 5,87 & 5 \\
\hline 36 & Kr83 & $22(\mathrm{nn}) 18(\mathrm{pp}) 3 \mathrm{n}$ & 5,6 & 55 & 45 & Lex18/24 & 4,61 & 4,23 & 8 \\
\hline 36 & Kr84 & 24(nn)18(pp) & 4,6 & 41 & 29 & & 5,02 & 4,84 & $9-6$ \\
\hline 36 & Kr85* & $23(\mathrm{nn}) 18(\mathrm{pp}) 3 \mathrm{n}$ & 5,9 & 56 & 44 & Lex18/24 & 4,98 & 4,25 & 8 \\
\hline 37 & Rb83* & $23(\mathrm{nn}) 17(\mathrm{pp}) 3 \mathrm{p}$ & 7,7 & 46 & 20 & In $4 / 15$ & 3,45 & 6,09 & 5 \\
\hline 37 & Rb84* & 23(nn)18(pp)np & 5,2 & 50 & 32 & In $3 / 8$ & 5,13 & 6,21 & 8 \\
\hline 37 & $\mathrm{Rb} 85$ & $24(\mathrm{nn}) 17(\mathrm{pp}) 3 \mathrm{p}$ & 6,3 & 46 & 20 & In $4 / 15$ & 3,44 & 6,13 & 5 \\
\hline 37 & $\mathrm{Rb} 86$ & 24(nn)18(pp)np & 5,3 & 51 & 30 & In $3 / 8$ & 5,07 & 6,23 & 8 \\
\hline 37 & $\mathrm{Rb} 87$ & $25(\mathrm{nn}) 17(\mathrm{pp}) 3 \mathrm{p}$ & 5,9 & 43 & 25 & In $3 / 18$ & 4,49 & 5,77 & 6 \\
\hline
\end{tabular}


Note: *) - the designation of unstable isotopes; 1$)$ the values $k_{1}-k_{2}$ refer to the radii $r_{n n}$ and $r_{p p}$, respectively.

It should be noted that the estimates of $r_{e}$ depend on the theoretical model of the charge distribution in the nucleus and the mechanism of interaction of the probing particles with the nucleus [5]. As a rule, a model is used in which the charge density decreases from one to zero exponentially in the surface layer of the nucleus with a thickness $t \sim 2.3 \mathrm{Fm}$ (Fig. 4). This layer can be correlated with the EF flows forming the Kelvin oval. The charge distribution in OSH with the outer Ex was similar to the charge distribution in $n[8,15]$ (Fig. 4).

The Kelvin oval is formed mainly by EF flows generated by external OSH and CSH. These flows, in conjunction with the flows of internal CSF EFs, bind the vortex structure of the nucleus into a single whole, that is, they play the role of nuclear forces. Outside the Kelvin oval at distances greater than $\sim 2 r_{e}$, the effect of its fluxes on the external medium's EP is manifested by the power lines of the electric field of the nucleus. It can be assumed that the configurations of this field will be sensitive to the types of external OSH and CSH and this will affect the energy and shape of the electron orbitals of the atom.

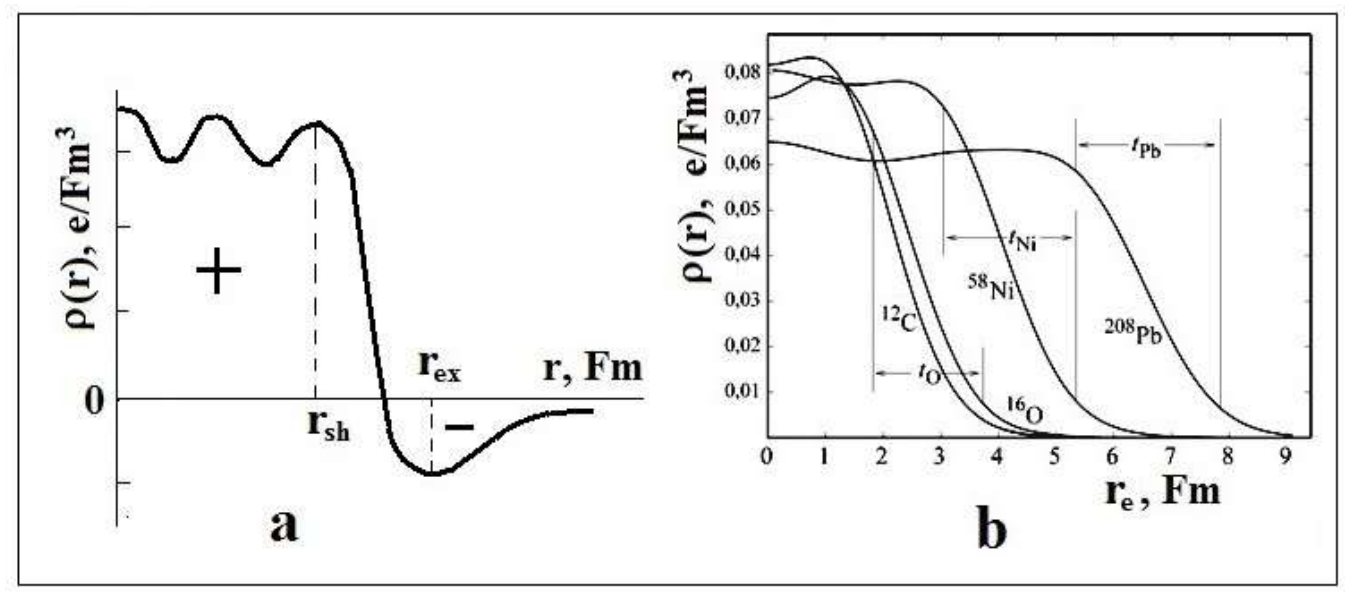

Fig. 4. Distribution of charge density in nuclei: (a) - vortex model, (b) - electron scattering [11]. The thickness of the surface layer is $t \sim 2.4 \mathrm{fm}$.

The proposed structure of external $\mathrm{OSH}$ and $\mathrm{CSH}$ correlates with the energy of separation of $\mathrm{n}$ and $\mathrm{p}$ from the nucleus, as evidenced by the data in Table. 1. In addition, calculations of the size and sign of the quadrupole moment of the outer shell allow modeling the dynamics and shape of the Kelvin oval.

It is obvious that these characteristics of the nucleus influence the geometry of its electric field, and hence the configuration of the electron shells of the atom, on which their chemical properties depend. Indeed, a comparison of the sizes of nuclei and atoms showed a correlation for the elements at the beginning and end of the periods in the $\mathrm{Z}$ sequence (Fig. 5). 


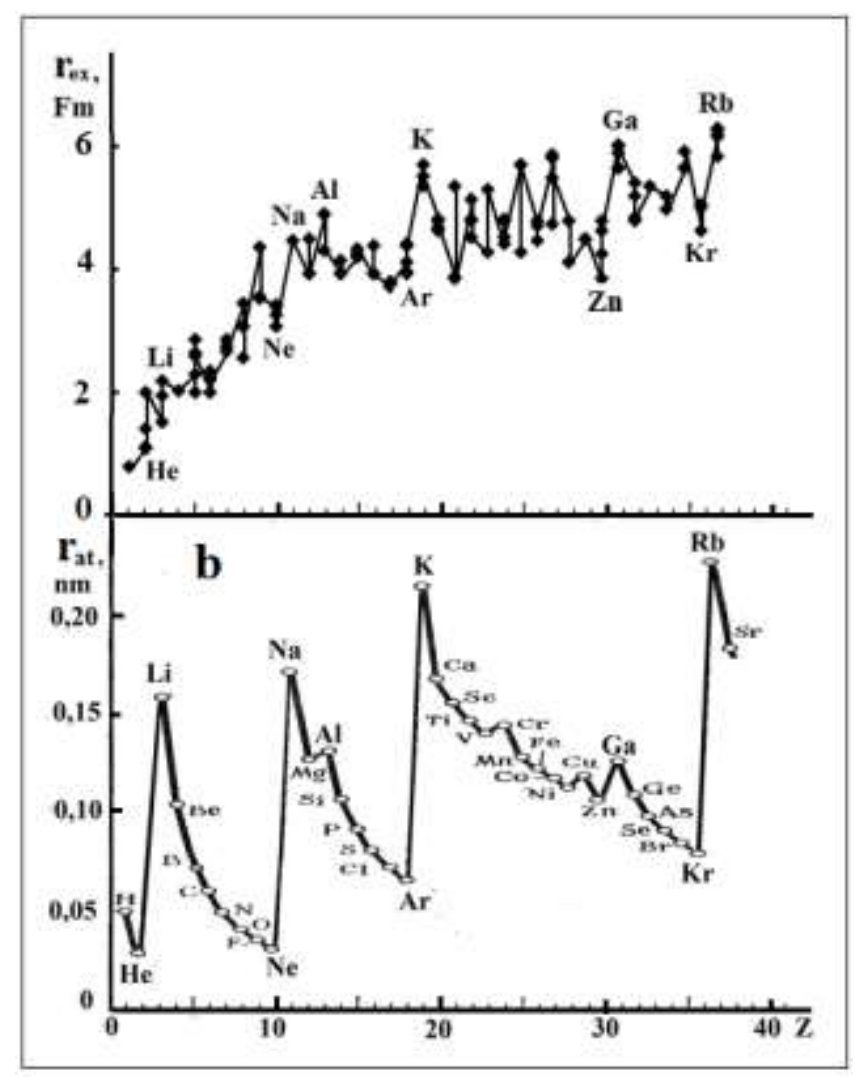

Fig. 5. Dependences of the radii of the nuclei (a) and the orbital radii of the atoms (b) on the ordinal number $(Z)$.

Thus, it can be concluded that the vortex structure of the nucleus makes a definite contribution to the formation of the electron shells of atoms and the regularities in the change in the types of outer shells and the size of the nuclei in sequences of the ordinal number manifest themselves on Mendeleyev's periodic law.

\section{CONCLUSIONS}

1. Within the framework of the laws of classical quantum mechanics and vortex motion, a model of the structure of the nucleus is constructed, consisting of nested shells formed from spherical and annular vortices.

2. It is shown that the structure and type of the outer shell of the nucleus basically determine: the energy of detachment of the first nucleon from the nucleus, its spin, magnetic moment, shape and size.

3. Rules for the formation of the outer shell of the nucleus, taking into account the empirical characteristics of the nuclei as boundary conditions, made it possible to calculate the frequency of the shell spin, the mass distribution, and the radius. 
4. The correlation between the dependences of the radii of the nucleus and the atom on the order number $\mathrm{Z}$ indicates the determinacy of the periodic Mendeleyev law by the regularity in the formation of the vortex structure of the nucleus.

\section{REFERENCES}

1. Elyashevich MA, Periodic Law of the D.I. Mendeleyev, spectra and atomic structure, UFN, -1970. -T.100. -Vyp.13. -FROM. 6-43.

2. Kholmanskiy A., Activation energy of water structural transitions. // Journal of Molecular Structure. (2015) 1089.-R. 124-128.

3. Zakharov $\mathrm{SD}$, Ortho / pair of spin-isomerism of $\mathrm{H} 2 \mathrm{O}$ molecules as the leading factor in the formation of two structural motifs in water. [Electronic resource]. Access mode: http://www.biophys.ru/archive/h2o-00025.pdf.

4. Gangrsky Yu.P., Markov BN, Nuclei in the laser beam. M .: Knowledge, 1984. -64 p.

5. DV Sivukhin, General course of physics. Volume 5. Atomic and nuclear physics. Moscow: Fizmatlit, 2002. - 784 p.

6. Kholmanskiy A.S. Fractal-resonant principle of action // MIS-RT-2003. Collection No. 29-

2. URL: http://www.ikar.udm.ru/sb/sb29-2.htm

7. Kholmanskiy A.S. Chirality and quantum effects as factors of morphogenesis //

Mathematical morphology. Electronic mathematical and medico-biological journal. - Vol. 9. Issue. 4. - 2010. - URL: http://www.smolensk.ru/user/sgma/MMORPH/N-28html/kholmanskiy-2/kholmanskiy-2.htm.

8. Kholmanskiy A.S. Vortex models of an electron and nucleons // Ibidem. - 2011.-T.10. Vyp.2. URL: http://www.smolensk.ru/user/sgma/MMORPH/N-30-html/kholmanskiy3/kholmanskiy-3.doc.

9. Physical quantities: Handbook. - Moscow: Energoatomizdat, 1991. - 1232 p.

10. Varlamov VV, Goncharova NG, Ishkhanov BS, Nuclear Physics and Nuclear Data Banks: A Training Manual. -M .: University Book, 2010. -246 p.

11. R. Hofstadter, The structure of nuclei and nucleons, UFN. -1963. -T.81. -FROM. 185-200.

12. Wang L.-B., Müller P., Laser Spectroscopic Determination of the 6He Nuclear Charge Radius // Phys. Rev. Lett. 93, 2004. 142501.

13. Müller P. et al. Nuclear Charge Radius of 8He // Phys. Rev. Lett. 2007. 99, 252501.

14. Makhankov VG, Rybakov Yu.P., Sanyuk VI, Skyrme Model and Strong Interactions (To the 30th Anniversary of the Creation of the Skyrme Model), UFN. 1992, №2. P.1-61 
15. Nedorezov VG, Mushkarenkov AN, Electromagnetic interactions of nuclei // URL: http://nuclphys.sinp.msu.ru/eint/index.html. 